\title{
Restaurierung eines Spielsteinkastens der Württembergischen Landesbibliothek
}

\section{Koordinierungsstelle für die Erhaltung des schriftlichen Kulturguts (KEK)}

Die Konferenz der Leiterinnen und Leiter Archivverwaltungen des Bundes und der Länder (KLA), die Kommission für Bestandserhaltung des Deutschen Bibliotheksverbands (dbv) sowie die Bundeskonferenz der Kommunalarchive beim Deutschen Städtetag (BKK) befassen sich seit den 1990er Jahren auf Bundesebene mit Fragen zur Bestandserhaltung in Archiven und Bibliotheken. Die Koordinierungsstelle für die Erhaltung des schriftlichen Kulturguts KEK wurde am 1. Juli 2011 an der Staatsbibliothek zu Berlin - Preußischer Kulturbesitz eingerichtet.

Die KEK unterstützt bundesweit ausgewählte Vorhaben, die sich mit der Erhaltung von schriftlichem Kulturgut befassen und innovativ, öffentlichkeitswirksam und modellhaft sind. Von 2010 bis 2015 wurden rund 2,4 Mio. Euro für Modellprojekte, die unter einem bestimmten Thema stehen, zur Verfügung gestellt. Die Bestände, die in der "Pilotphase" gesichert werden konnten, sind in ihrer Vielfalt in der Publikation "Schrift. Gut.Schützen." eindrucksvoll dokumentiert.

\section{KEK-Modellprojekt 2016: Erste Wahl}

Im Februar 2016 erfolgte der Aufruf für die KEKModellprojektförderung "Erste Wahl“, an dem sich die Württembergische Landesbibliothek mit dem Antrag "Restaurierung eines Spielsteinkastens in
Puzzleform aus dem 18. Jahrhundert - einzigartiges Lernmaterial und Württembergisches Pflichtexemplar" beteiligte. Im Juli 2016 traf die Vorabankündigung auf finanzielle Unterstützung in Höhe von knapp $4.000 €$ ein, die Mittelfreigabe erfolgte im August. Die Restaurierung, Konsolidierung und Verpackung der Medienkombination war Ende November 2016 abgeschlossen.

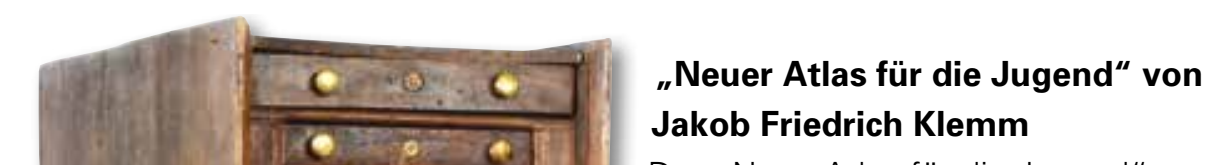

Der "Neue Atlas für die Jugend“ erschien 1782 im Tübinger Verlag Jacob Friedrich Heerbrandt. Autor war Jakob Friedrich Klemm, der im Vorwort "Veranlaßung zu der neuen Erfindung des kleinen Atlas für Kinder" ausführt: "Ich gebe hier der Jugend eine neue Erfindung von Land=Karten in die Hände, welche ihnen die Kenntniß unseres Erdbodens nicht nur angenehm machen, sondern auch erleichtern solle." Weiter schreibt der Vater von mehreren Kindern: "Man ist in unseren Tagen ganz davon überzeugt, daß die Erdbeschreibung keinen geringen Theil der nöthigen Kenntnisse eines vernünftigen Menschen ausmacht" und sieht darin einen Weg „die jungen Erdenbürger aus ihrer kleinen Ecke herausdenken" zu lehren.

Der Atlas in Oktavformat enthält die ausführliche Beschreibung der Erdteile und europäischen Länder, so wie sie zu Hölderlins Zeit bekannt waren. Demnach seien die Schweizer „treu, aufrichtig, tapfer", die "Chineser wizig, ehrerbietig gegen ihre Eltern und Lehrer, höflich, arbeitsam, sparsam, weichlich, geizig, untreu, betrügerisch... Sie haben Gelehrte, machen das feinste Porcellan, 
Lakkierarbeiten und Seidenstoffen" usw. „Außer den fünf Karten von den Welttheilen Europa, Asia, Afrika, Amerika und Süd-Indien, dem fünften und erst neu entdekten Welttheilem sind [dem Atlas] noch fünfzehn Specialkarten von den europäischen Reichen, und eine von Palästina in Asien...", insgesamt 21 Landkarten beigefügt. Diese „Kärtchen“ hätte Klemm "gerne den kleinen Erdenbürgern so in die Hände gegeben, daß sie dieselbige gleich hätten gebrauchen können." Doch aus Kostengründen ist dies nicht möglich und Klemm gibt genaueste Anweisungen, wie die Karten von den Vätern und Lehrern auf Papier geklebt „und dieses von dem Kinde mit den Nahmen, die der Karte fehlen, und den Merkwürdigkeiten des Landes beschrieben" werden sollen. Damit gibt er Vätern (wo bleiben die Mütter?) und Lehrern eine Medienkombination an die Hand. Der Zweck ist, „noch vorhandene Schwierigkeiten, die den Kindern Geographie schwer und verdrüßlich machen, nach und nach zu entdecken."

Das Exemplar der Württembergischen Landesbibliothek ist eine Luxusausführung. Der kleine Textband ist in Kalbsleder gebunden und mit Gold verziert. Die beigefügten 21 Landkarten sind handkoloriert, entlang den Ländergrenzen in Puzzleteile geschnitten, auf der Rückseite sorgfältig mit schwarzer Tinte in einer winzigen Handschrift beschriftet und werden in den Schubladen des dazugehörigen Spielsteinkastens aufbewahrt. Der Kasten in Überformat hat die Form eines Buches, ist aus fein gemasertem Kirschbaumholz gefertigt und mit Messingknöpfen versehen. An beiden Seiten ist Platz für ein Spielbrett, dessen Herstellung Klemm genau beschreibt und auf das die Puzzles gelegt werden können. Es ist nur noch ein Spielbrett erhalten.

Die Luxusausführung des „Neuen Atlas für die Jugend" gelangte 1920 als Geschenk des Herrn Privatier Rud. Anselm, Stuttgart, in den Bestand der WLB. Sie ist unter der Signatur Ra 18 Kle 1 (Text) und 2 (Spielsteinkasten) verzeichnet. Eine andere Ausgabe ist im Katalog der WLB nicht nachgewiesen, auch nicht die "Zweyte vermehrte und verbesserte Auflage" von 1790.

Weitere Exemplare sind zwar in sechs deutschen Bibliotheken, in Dänemark, Österreich, Schweden, der Schweiz und Ungarn zu finden, jedoch ausschließlich als Textband, nicht als „Medien-

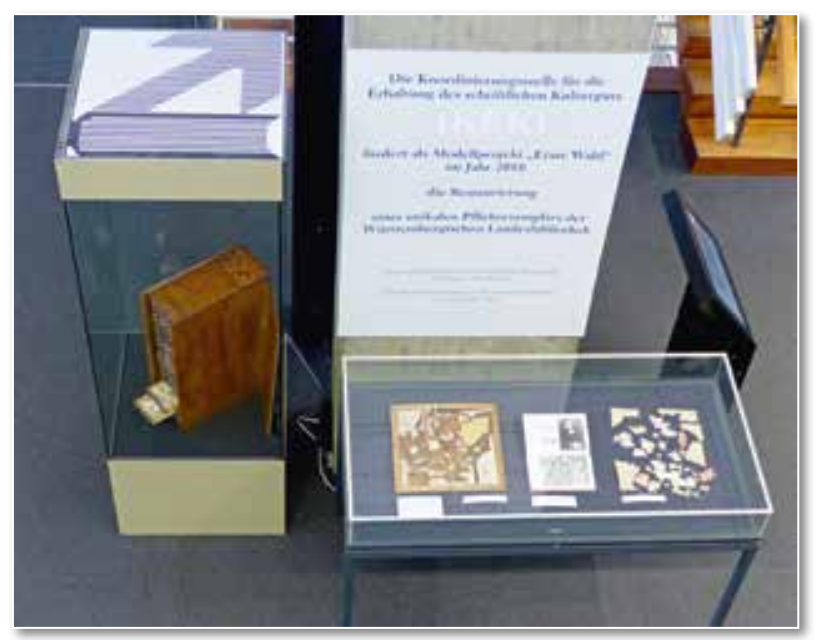

kombination" und zum Teil unvollständig. Bemerkenswert aber ist, dass das Stadtarchiv Karlsruhe ein Exemplar als Medienkombination besitzt. Es wird dort als Erdkunde-Puzzle des 18. Jahrhunderts geführt, besteht aus einem Miniatur-Sekretär aus Pappe und gelangte 1956 als Geschenk an das Haus Solms in den Bestand. Absenderin war Frau Helene Klemm, Rendsburg, eine Ururenkelin Jakob Friedrich Klemms.

\section{Jakob Friedrich Klemm}

Jakob Friedrich Klemm, am 25. August 1733 in Herrenberg geboren, hatte nach den Ausführungen seiner Urenkelin Johanna Elisabeth Roos einen "lebhaften Geist und eine ungewöhnliche Begabung". Er lernte schon als Vierjähriger ohne weitere Anleitung lesen, was die Eltern bewog, den zarten Buben "in Zucht und Vermahnung zum Herrn zu erziehen" und Theologie studieren zu lassen. Jakob Friedrich Klemm besuchte die Klosterschulen in Denkendorf und Maulbronn. 1752 begann er in Tübingen mit dem Studium der Theologie, während dem er zu der Erkenntnis kam, „dass für Lehrer der Religion die Kenntnis der Natur unentbehrlich sei" und das er 1757 mit Auszeichnung abschloss. Danach nahm er Anstellungen als Hauslehrer und Stiftsrepetent in Tübingen an, wo "sich ihm die richtigen Grundsätze für Erziehung und Unterricht immer heller ins Licht stellten, die sich hernach bei so vielen Zöglingen erproben sollten."

1763 versetzte ihn das herzogliche Konsistorium auf die Helferstelle in Balingen. Dort bemühte er sich um die einfache Sprache des Volkes. Seine Urenkelin überlieferte, dass Klemm zu der Erkenntnis kam, "sollte die Predigt einen ausgebreiteten Nutzen schaffen als bisher, so mußte die Schule, diese Pflanzstätte künftiger Väter und 


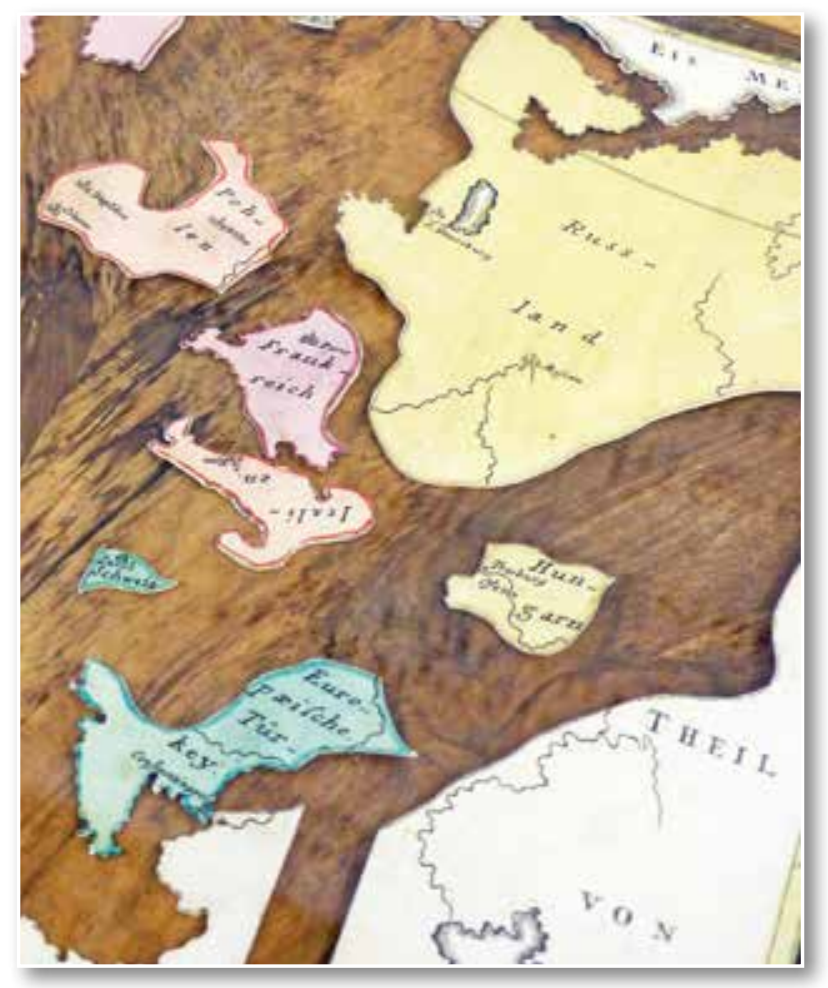

Mütter, von Grund aus umgestaltet ... werden." In Balingen machte Klemm die Bekanntschaft mit dem Züricher Pfarrer Lavater, veröffentlichte 1769 die „Exempel-Bibel zur Bildung des Herzens junger Leute und Kinder" mit von inm selbst verfassten kindgemäßen Liedern und bereitete den „Neuen Atlas für die Jugend" vor, eine Medienkombination für Unterrichtsdidaktik, die 1782 bei Heerbrandt in Tübingen erschien. Das Werk widmete er Herzog Carl Eugen von Württemberg, der inn vielleicht deswegen noch im gleichen Jahr als Dekan nach Nürtingen versetzte.

Dort gründete Klemm die erste Real- und Bürgerschule Württembergs. Diese "Nebenschule" sollte Lehrlinge fortbilden, für Schüler der oberen deutschen Schulklassen offen sein und Lehrer für die deutsche Landschule erziehen. Der Lehrplan enthielt Sachkunde mit Rechnen, Geometrie und Zeichnen, Naturgeschichte und Geschichte, Landwirtschaft und Botanik sowie die Erdbeschreibung: "Weil es ... Pflicht der Handwerksburschen ... ist, in die Fremde zu gehen, so sollen sie vorher die Erde kennen lernen" sowie "Französisch soviel, daß sie sich auf der Wanderschaft ein bißchen ausdrücken ... können."

1790 gab Superintendent Klemm die „Zweyte vermehrte und verbesserte Auflage" des "Neuen Atlas für die Jugend" heraus, die in der Allgemeinen Literatur-Zeitung lobend besprochen wurde. 1791 verfassten Klemm und Nathanael Köstlin, der zweite Pfarrer in Nürtingen, Empfehlungsschreiben, mit denen Friedrich Hölderlin dem Züricher Pfarrer Johann Caspar Lavater einen Besuch abstatten konnte. Die Begegnung Lavaters mit Hölderlin wurde für beide Seiten denkwürdig: Lavater versah Hölderlins Eintrag in sein Fremdenbuch mit dem Zusatz "N[ota] B[ene]".

Am 24. Juni 1793 verstarb Jakob Friedrich Klemm in Nürtingen.

\section{Restaurierung des „Neuen Atlas für die Jugend“}

Die Medienkombination wurde als KEK-Modellprojekt 2016 restauriert, das Dipl.-Restauratorin Sonja Brandt in der Württembergischen Landesbibliothek betreute. Die Arbeiten am Spielsteinkasten führte Holzrestaurator Andreas Mattes, Sachsenheim, durch, die Konsolidierung des Kalbslederbands übernahm die Firma Norbert Schempp, Kornwestheim, ebenso die Anfertigung eines neuen Aufbewahrungsbehältnisses aus Wellpappe.

Die Materialität, den Schadensbefund vor der Restaurierung sowie das Restaurierungskonzept des Spielsteinkastens beschreibt Andreas Mattes in seiner Dokumentation zur Restaurierung und belegt sie durch zahlreiche Fotodokumentationen. Das Ergebnis seiner Maßnahmen ist überaus erfreulich. Der Spielsteinkasten erstrahlt in neuem Glanz durch die Oberflächenreinigung, die Entfernung der Schmutzschichten und das Festigen und Ergänzen fehlender Substanzen. Dies gewährleistet zudem erst eine zukünftige Nutzung.

Das KEK-Modellprojekt 2016 wurde im Rahmen der Weihnachtsausstellung in der Landesvertretung Baden-Württemberg in Berlin vorgestellt. Seit Januar 2017 sind der Spielsteinkasten, der Textband und einige Landkarten in einer kleinen Ausstellung in der WLB zu sehen. Erläuterungen zum gesamten Themenkomplex in der WLB können auf einem Terminal angesteuert werden.

Vera Trost

Literatur:

Aus den Papieren einer schwäbischen Familie. Aufzeichnungen der Vorfahren der Familie Klemm, gesammelt von Johanna Elisabeth Roos. Calw \& Stuttgart: Verlag der Vereinsbuchhandlung 1898. Calwer Familienbibliothek, 45. Band.

450 Jahre Kirche und Schule in Württemberg. Katalog der Ausstellung, Stuttgart 1984, S. $142 \mathrm{ff}$.

Breymayer, Reinhard: Ein Porträt von Hölderlins Nürtinger Dekan Jakob Friedrich Klemm, in: Suevica. 7. Beiträge zur schwäbischen Literatur- und Geistesgeschichte, Stuttgart 1993, S. 82-113.

Mattes, Andreas: Dokumentation zur Restaurierung "Neuer Atlas für die Jugend“. J. F. Klemm 1782, WLB Stuttgart Ra 18 Kle1-2, Vaihingen an der Enz, 2016. 